\title{
LA NECESIDAD DE UNA POLÍTICA PÚBLICA PARA EL DESARROLLO DE SISTEMAS INTEGRADOS DE TRANSPORTE EN GRANDES CIUDADES MEXICANAS ${ }^{1}$
}

Alfonso Iracheta Cenecorta ${ }^{2}$

\section{El fenómeno del transporte urbano en México: problemas y perspectivas}

\section{EL TRANSPORTE URBANO EN MÉXICO: ENTRE LA MOVILIDAD Y LA CRISIS}

El PROBlema de LA MOVILIDAD dE LAS PERSONAS EN LAS CIUDADES MEXICANAS

En el año 2000, 65.7 millones de mexicanos vivían en 364 ciudades y el grado de urbanización alcanzó $67.3 \%$. Como consecuencia, se ha transforma-

1 Este texto es una síntesis apretada de varios capítulos del libro de IRACHETA, Alfonso (2006): La necesidad de una Política Pública para el Desarrollo de Sistemas Integrados de Transporte en Grandes Ciudades Mexicanas. El Colegio Mexiquense y otras instituciones, Zinacantepec, México. do el proceso de urbanización de preeminente a policéntrico, y de metropolitano a megalopolitano -regiones urbanas-, sin modificar en su esencia la estructuración desigual del espacio ${ }^{3}$.

Hoy, movilizarse diariamente es un asunto prioritario para la producción y la productividad, para la seguridad física y emocional de las personas y para el desarrollo de casi todas las funciones humanas.

Por ello, las actividades de la población y su ubicación en las grandes ciudades y zonas metropolitanas generan la necesidad de una permanente y creciente movilidad y es responsabilidad de las autoridades facilitar, por medio de la ordenación de los usos del suelo, la creación de infraestructu-

2 México. Arquitecto; Maestro en Planeación Urbana, Universidad de Edimburgo; Doctor en Estudios Regionales Universidad de Varsovia, Polonia. Coordinador del Programa Interdisciplinario de Estudios Urbanos y Ambientales (PROURBA) de El Colegio Mexiquense.

Correo electrónico:mvarela@cmq.edu.mx

3 Garza, G. 2003. 
ras y la organización de los desplazamientos que se realizan en transporte público y en automóviles.

La separación física entre las distintas funciones urbanas, la necesidad creciente de comunicarse por parte de los ciudadanos y la falta de una disciplina para ordenar y desarrollar las redes viales y las del transporte público han tenido como consecuencia, por una parte, una tendencia a la reducción en la capacidad de movilización de las personas, y, por la otra, una disminución de su acceso a los servicios e infraestructura que la ciudad brinda.

El tema, entonces, para la política pública, es definir un proyecto urbano incluyente, que genere las condiciones de accesibilidad de personas y bienes a sus lugares de destino como objetivo prioritario.

Las cifras del gobierno federal mexicano ${ }^{4}$ muestran la necesidad de casi duplicar en 25 años el parque de vivienda del país (de 22 a aproximadamente 41 millones de unidades). La pregunta es: ¿dónde se van a localizar esas viviendas? ¿Se hará como hasta ahora, predominando la irregularidad y el precarismo? ¿Las personas se movilizarán en vehículos públicos de baja capacidad y calidad -combis y micros- como los de ahora?

4 CONSEJO NACIONAL DE LA POBLACIÓN (CONAPO), 2000.

\section{EL PAROUE AUTOMOTOR Y LOS VIAJES EN LA CIUDAD}

De acuerdo con el gobierno federal ${ }^{5}$, el parque vehicular en México ha crecido de manera constante. Se estima que en el país existían en 2003 poco más de 21.2 millones de vehículos, destacando los automóviles particulares, que hacen un total de 14 millones. Asimismo, anualmente se incorporan 1.16 millones de nuevos vehículos, con tasas anuales de $7.45 \%$, las cuales son muy superiores a las demográficas (cercanas a 2\%) y al crecimiento de la economía (del orden de 4\%), por lo que debe llamar la atención esta dinámica debido a las consecuencias que tiene en la acumulación de vehículos, en la baja capacidad de producir infraestructura vial para que éstos circulen, y en la creciente relación que tiene el vehículo individual frente al colectivo. La gran mayoría de los nuevos vehículos son automóviles privados y se utilizan para movilizar a las personas dentro de las ciudades, además del tráfico interurbano.

Destacamos este fenómeno en la Zona Metropolitana del Valle de México ( $\mathrm{zmvm}^{6}$ ), aunque es claro que ya está presente de manera creciente en el resto de las ciudades del país. En efecto, la capital

5 INSTITUTO NACIONAL DE ESTADÍSTICA Y GEOGRAFÍA (INEGI), 2000; INSTITUTO NACIONAL DE ESTADÍSTICA Y GEOGRAFÍA (INEGI), 2004; INSTITUTO NACIONAL DE ESTADÍSTICA Y GEOGRAFÍA (INEGI), 2005.

6 La delimitación de la ZMVM atiende a diversos criterios, por lo que los datos pueden diferir en algunos casos, de acuerdo con la fuente de información consultada. transporte en grandes ciudades mexicanas / Alfonso Iracheta Cenecorta 
nacional y su zona conurbada concentran aproximadamente 25\% del parque vehicular nacional, aunque su población tiene una proporción menor (del orden de 19\%). Sólo en esta metrópoli, el parque vehicular se incrementó de 3.28 millones en 1997 a 4.45 en $2001{ }^{7}$ destacando que solamente $1.31 \%$ de los vehículos eran camiones de pasajeros (oficiales y particulares), mientras que los coches particulares representaron casi 85.2\%.

Se estima que en la zmvm se realizan alrededor de 35 millones de viajes/persona/día, lo que significa que cada habitante efectúa aproximadamente 1.8 viajes diariamente (1.78). ${ }^{8}$ De éstos, $78 \%$ (2003) se hace en transporte público, destacando los "taxis colectivos" (micros y combis) con 60\%. Los camiones de pasajeros - públicos y privados- en esta metrópoli representaron $1.31 \%$ del parque vehicular registrado en 2001, lo que da una idea de la proporción entre viajes y vehículos utilizados por la población ${ }^{9}$.

Es importante observar que mientras los modos de transporte en combis y micros mantienen tendencias a la alza (en 1994 representaban 54\%), los otros modos de transporte público (metro, tren li-

7 Dato obtenido considerando la delimitación de la ZMVM (Sedesol, Conapo, inegi, 2004), que se constituye de 59 municipios del Estado de México, las 16 delegaciones del Distrito Federal y un municipio de Hidalgo (Tizayuca).

8 Dato obtenido considerando la población total de la zmvm al año 2004 (19.680.616 habitantes).

9 NEGRETE, María Eugenia (coord.), 2005. gero y autobús) decaen, aunque paradójicamente estos últimos sean considerados más adecuados desde todos los puntos de vista, excepto por la flexibilidad para adecuarse a cualquier ruta. Los primeros, por el contrario, pueden acceder a cualquier lugar, aunque carezcan de la infraestructura para ello, y hacer paradas en cualquier punto, no obstante que al mismo tiempo sean los más inseguros y desordenados.

Como consecuencia de lo anterior, crece el uso del coche particular, que pasó de 17.4 a 20\% del total de viajes entre 1994 y 2003 en la zmvm, con lo cual pudiera asumirse que la lucha por un transporte público masivo de calidad y ambientalmente sustentable se está perdiendo en la zmvm, y, como muestran las evidencias que dan a conocer los expertos de otras ciudades (Monterrey, León, Tijuana, Querétaro, Puebla), es claro que el fenómeno -con más o menos dramatismo- se reproduce en la mayoría de las grandes ciudades de México.

El Fideicomiso de Estudios Estratégicos sobre la Ciudad de México (2000: 202-203) ${ }^{10}$ estimó que el parque vehicular de la zmvm alcanzaría cerca de 4.7 millones de vehículos en 2010 y 5.6 en

10 FIDEICOMISO DE ESTUDIOS ESTRATÉGICOS SOBRE LA CIUDAD DE MÉXICO, 2000. 
2020, correspondiendo a automóviles particulares aproximadamente 4.2 (89.4\%) y 5.0 millones (89.3\%), respectivamente.

Lo que los datos muestran es una clara tendencia que tiene las siguientes características:

- El uso del coche particular es creciente y deriva de una cultura individualista y de una industria en expansión, que no considera las necesidades de la comunidad, de la ciudad y del ambiente. En todas las ciudades, la tasa de motorización crece por encima de la del transporte público, e incluso por encima de la población. Al no existir una estrategia proporcional y paralela de construcción de vialidades, es posible imaginar en el futuro la casi paralización del tránsito urbano por periodos cada vez más prolongados, hasta límites que pueden llevar al desquiciamiento social por las consecuencias en la economía, las relaciones sociales y la salud física y emocional de la mayoría de los ciudadanos.

- La carencia de infraestructura de transporte masivo adecuada y de un servicio de transporte público de calidad, ha llevado al sector de la población con un nivel de ingreso que les per- mite adquirir y mantener un vehículo privado, a optar por su uso como un mal necesario.

- Los habitantes urbanos de menores ingresos no tienen más opción que hacer uso del transporte público porque el costo es más compatible con su nivel de ingreso. Así, deben convivir con las deficiencias de ese transporte, movilizándose predominantemente en vehículos de servicio público de baja capacidad y poco seguros, con una mala calidad del servicio y alto riesgo de accidentes y asaltos.

Mientras que en 1995 las ventas de vehículos en el país fueron de casi 188 mil unidades, en 2003 alcanzaron más de 990 mil, destacando los automóviles con prácticamente $70 \%$ y los camiones ligeros con $28.4 \%^{11}$

Finalmente, la información muestra que la producción automotriz está rebasando la capacidad de las ciudades para recibir más vehículos, por lo que esta tendencia generaría una situación crítica y paradójica que se podría enunciar así: cada día es posible producir automóviles más baratos y con financiamiento ampliado a mayores estratos de la población, pero no existe una infraestructura adecuada para recibir y permitir que circulen esos vehículos, con lo cual se diluye su sentido esencial;

11 INSTITUTO NACIONAL DE ESTADÍSTICA Y GEOGRAFÍA (INEGI), 2002; INSTITUTO NACIONAL DE ESTADÍSTICA Y GEOGRAFÍA (INEGI), 2005 
es decir, ofrecer más comodidad, seguridad, velocidad y precios óptimos para la movilización de las personas en las ciudades.

Frente a esto, destaca que en la zmvm y en general en las grandes ciudades, los vehículos de transporte público realizan entre 80 y $85 \%$ de los viajes/ persona/día, con tan sólo entre 7 y 12\% del parque vehicular.

De acuerdo con el Instituto Nacional de Ecología (INE) (1995: 26-27), las pérdidas económicas y de bienestar social en la zmvm derivadas del uso irrestricto del automóvil, ascendieron entonces a siete mil millones de dólares anuales. Igualmente, ya en 1992 el Banco Mundial estimó que el costo anual de la contaminación del valle de México en la salud de sus habitantes alcanzó alrededor de 70 mil millones de dólares, calculado como efectos en la morbilidad y mortalidad provocadas por partículas suspendidas, ozono y plomo. Qué sectores de la población son afectados mayormente por este problema es un asunto que requiere de análisis, para determinar hasta dónde la población que menos contamina es afectada en su salud.

Otros estudios (Fideicomiso de Estudios Estratégicos, 2000: 199) demuestran que año a año crece el número de viajes promedio en automóvil, al igual que su longitud, y que los ahorros en tiempo por el uso del automóvil se pierden por el incremento en los recorridos, ya que el tiempo promedio dedicado a desplazamientos vehiculares en la zmvm se estima entre 1.5 y 3.5 horas por persona al día. Cabe señalar que es la población más pobre la que dedica más tiempo para trasladarse, porque normalmente reside en la periferia y trabaja en las zonas centrales de la urbe, además de que tiene que trasladarse en sistemas de transporte ineficientes que multiplican los tiempos de traslado.

Frente a este fenómeno, las preguntas fundamentales que debe responder una política pública participativa y rigurosa son:

- ¿Qué se va hacer con los cerca de un millón 600 mil nuevos vehículos que se incorporan cada año a las ciudades del país? ${ }^{12}$

- ¿La creciente motorización de las ciudades mejora la calidad de vida de la población urbana o, por el contrario, incrementa la desigualdad social y la segregación socio-espacial de nuestras urbes?

- ¿Es posible imaginar que las carreteras urbanas (periféricos, viaductos, libramientos, ejes viales, circuitos interiores y exteriores) podrán ampliarse, y que esta política resolverá el problema de la movilidad de las personas y su accesibilidad a los destinos de la urbe, cuando todas las evidencias muestran que conforme se

12 Dato obtenido para el periodo 1995-2003. 
incrementan las vialidades, crece el parque vehicular en una espiral inacabable?

- ¿Es sustentable para las ciudades mexicanas seguir promoviendo la adquisición de coches particulares y manteniendo el "carcachismo/ chatarrismo"13, sin que proporcionalmente exista una política de movilización de las personas por transportes públicos?

\section{Conclusión}

México está pagando una onerosa factura directamente asociada con la falta de políticas integrales, participativas y de largo plazo para mejorar la movilidad urbana de personas y los transportes públicos y masivos. Lo anterior se ha visto reflejado:

- En los inmensos costos ambientales, que incluyen la contaminación de las cuencas atmosféricas e hidráulicas, la desaparición de grandes cantidades de suelo valioso para el adecuado funcionamiento de los ecosistemas, e incluso la alteración generalizada del clima que se agudiza en las ciudades.

- En el consumo excesivo de energéticos, que deriva de la necesidad creciente de alimentar los vehículos con hidrocarburos.

13 Mexicanismos: carcacha: coche viejo y destartalado; chatarra: basura, desechos generalmente de metal.
- En los cientos de vidas humanas que se pierden en accidentes automovilísticos.

- En los millones de horas-persona perdidas en embotellamientos carreteros y urbanos.

\section{¿Qué hacer?: Hacia una política nacional de transporte urbano}

Las líneas de política que enseguida se enuncian han sido desarrolladas en el libro que aquí se sintetiza:

\section{VISIÓN INTEGRAL DEL TRANSPORTE Y LA MOVILIDAD}

- La integración del transporte urbano como elemento fundamental de la planeación urbana.

- La reducción del número de automóviles particulares en circulación.

- La provisión de un sistema de transporte público de alta capacidad, eficiente, poco contaminante, seguro, cómodo y accesible.

- La promoción del uso de transporte no motorizado, como caminar y andar en bicicleta.

- El mejoramiento de tecnologías vehiculares y de la calidad de los combustibles.

OPINIÓN: La necesidad de una política pública para el desarrollo de sistemas integrados de transporte en grandes ciudades mexicanas / Alfonso Iracheta Cenecorta 


\section{LA IMPORTANCIA DE UN PROYECTO INTEGRAL Y PARTICIPATIVO}

- Promover la movilidad urbana incluyente como un derecho social.

- Integrar las políticas de movilidad, desarrollo urbano y de protección al medio ambiente.

- Modernizar el sector de transporte público urbano.

- Promover mejores condiciones de acceso para los ciudadanos a bienes y servicios esenciales, el trabajo, el hogar y la recreación.

- Promover y ampliar la seguridad y la calidad de vida por medio del aumento de la movilidad y de la accesibilidad de toda la gente, principalmente de aquellos con capacidades de movilidad diferentes o insuficientes.

- Incentivar la implantación de políticas para personas con restricción de movilidad, con la adaptación de los sistemas de transporte que tomen como principio el acceso universal a la ciudad como un derecho de todos.

- Apoyar la implantación de estrategias de seguridad vial, con la meta de reducir por lo menos en $50 \%$ el número actual de muertes por accidentes.

- Dar prioridad a la circulación, fluidez y orden en el transporte colectivo y el no motorizado, como estrategia para garantizar el crecimiento urbano sustentable, reducir sensiblemente la contaminación atmosférica y auditiva, y distribuir y adjudicar los espacios públicos de una manera más justa y democrática.

- Implantar medidas de reducción del uso del automóvil privado, principalmente en los centros urbanos y los corredores de mayor movilidad de personas, ofreciendo alternativas de transporte público.

- Incentivar la implantación de sistemas estructurales de transporte de capacidad media y alta en corredores de ciudades medianas y grandes y en las áreas metropolitanas.

- Aumentar las inversiones en el sistema vial urbano e interurbano que dé prioridad a los modos de transporte colectivo y no motorizado y que aproveche las plusvalías generadas en favor de la hacienda pública.

- Promover la transformación de la tarifa como instrumento de inclusión social y de la distribución equitativa de los bienes públicos.

- Estimular la participación ciudadana en la toma de decisiones corresponsable, que provenga tanto de la iniciativa popular como de la sociedad civil organizada, fomentando así el control social efectivo de las políticas públicas de movilidad. 


\section{INTEGRAR LAS POLÍTICAS DE MOVILIDAD, DESARROLLO URBANO Y PROTECCIÓN AL MEDIO AMBIENTE}

- Ligar las políticas públicas de transporte, movilidad y tránsito con la política de desarrollo urbano, con el objetivo de promover el desarrollo sustentable, colaborando en la formación y consolidación de centros suburbanos integrados a la ciudad.

- Estimular la adopción, en los planes de desarrollo urbano y ordenamiento territorial, de los principios de movilidad que resulten en una mejor distribución de las actividades en el territorio, utilizando el transporte colectivo como eje rector del desarrollo.

- Promover la mejora de la calidad ambiental urbana por medio del control del tránsito de vehículos motorizados, sobre todo en las áreas residenciales y de protección al patrimonio histórico, arquitectónico y cultural.

- Estimular la construcción de ciclovías, integrándolas como parte formal de la red de transporte público existente.

- Incentivar la producción y uso de vehículos que utilizan combustibles limpios y alternativos, integrando al proceso de diseño y ejecución de las políticas urbanas y de movilidad a las secretarías de transporte y energía, tanto del gobierno federal como de las entidades federativas.

\section{MODERNIZACIÓN, INTEgRACIÓN Y ESTRUCTURACIÓN DEL TRANSPORTE PÚBLICO URBANO}

- Planificar y programar la modernización del transporte público urbano a partir de la participación corresponsable de los actores sociales involucrados.

- Integrar los servicios y modos de transporte público en grandes sistemas urbano-metropolitanos.

- Desarrollar conceptos tecnológicos y logísticos en materia de vialidad y transporte y de suelo que incorporen la creación de corredores urbanos y no sólo rutas de transporte.

- Implantar modelos de solución de transporte masivo costo-efectivas.

- Reconocer que el transporte colectivo es un servicio público, por lo que la prestación de un servicio de calidad es responsabilidad del Estado.

- Asegurar que la participación de la iniciativa privada y de las organizaciones sociales en la prestación del servicio se dé dentro de un marco regulatorio que garantice que el usuario reciba un servicio de calidad.

- Facilitar procesos de estructuración de sistemas con mayor soporte técnico, financiero e institucional. 
- Buscar esquemas de financiamiento que incorporen recursos públicos y privados para mejorar los sistemas de transporte urbano-metropolitanos.

- Promover y desarrollar sistemas de transporte público tipo BRT (Bus Rapid Transit).

El conjunto de políticas exhibe la complejidad del fenómeno y la importancia de enfrentarlo de manera integral y con el compromiso público mostrado en otras políticas como la macroeconómica. La movilidad urbana dejó de ser un asunto marginal para transformarse en uno de los temas más relevantes de la política y la preocupación social.

\section{Bibliografía}

CONSEJO NACIONAL DE POBLACIÓN ICONAPO). Proyecciones de los hogares y viviendas 2000-2030, Total de hogares. México. 2000.

FIDEICOMISO DE ESTUDIOS ESTRATÉGICOS SOBRE LA CIUDAD DE MÉXICO. La Ciudad de México hoy, bases para un diagnóstico. Gobierno del Distrito Federal, México. 2000. P. 199 - 203. ISBN 968-816-299-X.

GARZA, Gustavo. La urbanización de México en el siglo XX. El Colegio de México, México. 2003. 101 p. ISBN 968-121-107-3.
INSTITUTO NACIONAL DE ECOLOGÍA (INE). Elementos de política ambiental para una ciudad sustentable: El manejo de la cuenca atmosférica. Documento de trabajo, borrador preliminar, mimeo, México. 1995.

INSTITUTO NACIONAL DE ESTADÍSTICA Y GEOGRAFÍA (INEGI). Anuario estadístico de los Estados Unidos Mexicanos. Aguascalientes, México. 2005. ISSN 0188-8692.

INSTITUTO NACIONAL DE ESTADÍSTICA Y GEOGRAFÍA (INEGI). Anuario estadístico de los Estados Unidos Mexicanos, edición 2004 (1995-2002). Aguascalientes, México. 2004. ISSN 0188-8692.

INSTITUTO NACIONAL DE ESTADÍSTICA Y GEOGRAFÍA (INEGI). Anuario estadístico de los Estados Unidos Mexicanos. Aguascalientes, México. 2002. ISSN 0188-8692.

INSTITUTO NACIONAL DE ESTADÍSTICA Y GEOGRAFÍA (INEGI). Estadísticas históricas de México (1925-1990). Aguascalientes, México. 2000. ISBN 970-13-2117-0.

NEGRETE, María Eugenia (coord.). Grandes temas sobre transporte, vialidad y movilidad en la ZMVM. Metrópoli 2025, Comité de Transporte, Vialidad y Movilidad. México. 2005.

SECRETARÍA DE DESARROLLO SOCIAL (SEDESOL), CONAPO, INEGI. Delimitación de las zonas metropolitanas de México. México. 2004. ISBN CONAPO 970-628-828-7. ISBN INEGI 970-133675-5.

revista invi № 71 / Mayo 2011 / Volumen № 26: 133-142 141 
SECRETARÍA DE MEDIO AMBIENTE Y RECURSOS NA-

TURALES (SEMARNAT). Inventario de emisiones de la ZMVM 2002 (versión preliminar para consulta en la página electrónica de la Semarnat: www.semarnat.gob.mx), México. 2002.

SECRETARÍA DE MEDIO AMBIENTE Y RECURSOS NATURALES, Instituto Nacional de Ecología (SEMARNAT, INE). El medio ambiente en México, 2002. Con base en información de la Dirección General de Investigación sobre la Contaminación Urbana, Regional y Global, México. 2002. 